\title{
Improved understanding of rice amylose biosynthesis from advanced starch structural characterization
}

\author{
Enpeng $\mathrm{Li}^{1}$, Alex Chi Wu${ }^{2}$, Juan $\mathrm{Li}^{3}$, Qiaoquan $\mathrm{Liu}^{3}$ and Robert G Gilbert ${ }^{1,2^{*}}$
}

\begin{abstract}
Background: It has been shown from the chain length distributions (CLDs) that amylose chains can be divided into at least two groups: long and short amylose chains. These molecular structures influence some functional properties of starch, such as digestibility and mouth-feel. GBSSI is the key enzyme for the elongation of amylose chains; however, the effect of other starch biosynthesis enzymes in amylose synthesis is still not fully understood. Two advanced starch characterization techniques, size exclusion chromatography (SEC) and fluorophore-assissted carbohydrate electrophoresis (FACE), together with a newly developed starch biosynthesis model, are used to improve understanding of amylose biosynthesis.
\end{abstract}

Results: SEC and FACE were used to determine the CLD of amylose and amylopectin in various native and mutant rice starches. The types of starch branching enzymes (SBEs) involved in the synthesis of the distinct features seen for shorter degrees of polymerization, DP, <2000, and longer (DP > 2000) amylose chains are identified by combining these data with a mathematical model of amylopectin biosynthesis. The model enables each feature in the amylopectin CLD to be parameterized in terms of relative SBE activities, which are used to explain differences in the genotypes.

Conclusions: The results suggest that while GBSSI is the predominant enzyme controlling the synthesis of longer amylose chains, some branching enzymes (such as BEl and BEllb) also play important roles in the synthesis of shorter amylose chains.

Keywords: Fluorophore-assisted carbohydrate electrophoresis; Size exclusion chromatography; Chain length distribution; Modeling; Amylose biosynthesis; Starch branching enzyme; Molecular structural characterization

\section{Background}

Starch is a homopolymer of glucosyl units, with two main components: amylose and amylopectin. Amylose is a slightly branched molecule, and amylopectin is highly branched (Buleon et al. 1998; Tester et al. 2004). Amylose molecules usually have 3 to 11 branches, and the average length of the chains from debranched amylose molecules is degree of polymerization (DP) 200 (Wang and White 1994; Yoshimoto et al. 2000). Amylopectin

\footnotetext{
* Correspondence: b.gilbert@uq.edu.au

${ }^{1}$ Tongji School of Pharmacy, Huazhong University of Science and Technology, Wuhan, Hubei 430030, China

${ }^{2}$ Centre for Nutrition and Food Sciences, Queensland Alliance for Agricultural and Food Innovation, The University of Queensland, Brisbane, QLD 4072,

Australia

Full list of author information is available at the end of the article
}

branches can be divided into three groups (A type, B type, and $C$ type) depending on their organization in the amylopectin clusters. A type branches are the outer chains which carry no branches, and their average length is DP 6-16 (Hizukuri 1985, 1986). B type branches are the chains carrying branches; $\mathrm{B}$ type branches can be divided into $B_{1}, B_{2}, B_{3}$, and $B_{4}$ chains depending on number of clusters traversed (Wang and White 1994; Hizukuri 1986; Mua and Jackson 1997). Each amylopectin molecule only has one $\mathrm{C}$ type branch, which has the reducing end.

Granule-bound starch synthase I (GBSSI) is the key enzyme for amylose biosynthesis. Two amylose synthesis models have been proposed based on in vitro experiments. 
One model suggests that malto-oligosaccharides (MOSs) act as the primer for amylose chain synthesis, and without an MOS one has longer amylopectin chains rather than amylose chains (Ball et al. 1998). The other model suggests that amylose synthesis starts with a high molecular weight primer inside the starch granule (Ball et al. 1998).

Although it is well known that elongation of amylose chains is predominantly by GBSSI and that amylose branches are formed by starch branching enzymes (SBEs) (Denyer et al. 2001; Ball et al. 1998), little is known about the details of how the individual isoforms of SBE and starch synthase (SS) contribute to the amylose chain-length distribution (CLD). The lengths, and the relatively few but significant numbers, of these chains influence functional properties such as digestion rate and location, and mouth-feel (Syahariza et al. 2013). Previous studies have shown that debranched amylose distributions have at least two distinct groups (Ward et al. 2006; Syahariza et al. 2013; Li et al. 2011; Witt et al. 2012), which are here referred to as the short and long amylose features. Their synthesis may be controlled by different enzyme combinations.

Previous studies on amylose synthesis are mostly based on in vitro experiments; the present study uses advanced starch structural analysis and interpretation techniques to give a new insight into in vivo amylose synthesis. Fluorophore-assisted carbohydrate electrophoresis (FACE) and size exclusion chromatography (SEC) are used to analyze the CLDs of amylopectin and amylose molecules in wild-type and transgenic rices, the transgenic lines lacking one or more SBEs. A model (Wu and Gilbert 2010; $\mathrm{Wu}$ et al. 2013) for the amylopectin biosynthetic process is used to fit the amylopectin CLD obtained using FACE to incorporate the underlying mechanism (enzyme activity information) of amylopectin synthesis. This activity information will be seen to aid understanding of the amylose biosynthesis mechanism by seeing which activity change in amylopectin is reflected in a change in amylose CLD. This provides a means to examine which starch-synthesis enzymes besides GBSSI affect the synthesis of the different amylose components.

\section{Results}

\section{CLDs of amylopectin}

The experimental and model-fitted CLDs of amylopectin from all rice samples obtained using FACE, normalized to their highest peaks, are shown in Fig. 1. The parameters in the model are given in the Methods section. BEI and BEIIb knockout WXJ rice amylopectin has relatively more long chains (DP $>15)$, as is reflected in larger $h_{(\mathrm{iii} / \mathrm{i})}$ and $h_{(\mathrm{v} / \mathrm{i})}$ values in the model fitting than those of the wild type WXJ rice (Table 1). The Wu-Gilbert model fitting result (Table 1) shows that the values of the most important parameters governing the shape of the amylopectin CLD, $\beta_{(\mathrm{ii})}, \beta_{\text {(iv) }}$ and $\beta_{\text {(vi) }}$ are not very different between both rice starches, while those of $\beta_{(\mathrm{i})}, \beta_{(\mathrm{iii})}$ and $\beta_{(\mathrm{v})}$ (and hence $\left.\gamma_{(\mathrm{i}, \mathrm{ii}}, \gamma_{(\mathrm{iii}, \mathrm{iv})}, \gamma_{(\mathrm{v}, \mathrm{vi})}\right)$ for wild-type WXJ rice starch are significantly larger than those of BEI- and BEIIb-knockout WXJ rice starch.

Koshihikari rice amylopectin has relatively more long chains $(\mathrm{DP}>30)$ than Daohuaxiang rice starch. Reflecting this, the values of $\beta_{(\mathrm{i})}, \beta_{(\mathrm{ii})}, \beta_{(\mathrm{iii})}, \beta_{(\mathrm{v})}, \gamma_{(\mathrm{i}, \mathrm{ii})}, \gamma_{(\mathrm{iii}, \mathrm{iv})}$ and $\gamma_{(\mathrm{v}, \mathrm{v})}$ are similar between the two rice lines, and Koshihikari has higher $\beta_{(\mathrm{iv})}$ and $\beta_{(\mathrm{vi})}$ values than Daohuaxiang. The value of $h_{(\mathrm{iii} / \mathrm{i})}$ of Koshihikari is higher than that of Daohuaxiang, while $h_{(\mathrm{v} / \mathrm{i})}$ of Koshihikari is lower.

\section{CLDs of amylose}

Amylose CLDs were obtained by universal calibration from SEC analysis; because of the limited calibration range, the amylose results cannot be quantitatively compared to those from FACE in the DP region where these overlap. Amylose CLDs are reported here in terms of apparent DP $X_{\text {app }}$, in cognizance of the limitations of the restricted calibration used here. SEC also suffers from band-broadening, which can mask subtle features (Castro et al. 2005). This section concerns only the amylose components, which can only be obtained from SEC, which, as just noted, suffers inaccuracies; those of amylopectin obtained from FACE, discussed in the preceding section, do not suffer from these inaccuracies, so the amylopectin component obtained from SEC is not discussed. The CLDs of amylose chains are normalized to their highest amylose peaks and are shown in Fig. 2 and Fig. 3. As seen elsewhere, e.g. (Ward et al. 2006; Syahariza et al. 2013; Li et al. 2011; Witt et al. 2012), if the SEC separation is sufficiently good, at least two distinguishable features are seen in rice amylose CLDs. We denote those seen here as the shortchain feature AM1, ranging from DP 150 to 2000, and the long-chain feature AM2, ranging from DP 2000 to 10000. $X_{\mathrm{AM} 1}$ and $X_{\mathrm{AM} 2}$ refer to the corresponding peak positions. The ratio of the AM1 area under peak (AUP, defined by the dividing lines on the appropriate figures) to whole-amylose AUP from wild type WXJ rice is larger than that from BEI \& BEIlb knockout WXJ rice, while the ratio of AM2 AUP to whole-amylose AUP shows the opposite (Fig. 2a). The $X_{\mathrm{AM} 1}$ and $X_{\mathrm{AM} 2}$ values of both wild type WXJ rice and BEI \& BEIIb knockout WXJ rice are similar (Table 1). The $X_{\mathrm{AM} 1}$ ratio of Koshihikari rice is larger than that from Daohuaxiang rice, while the ratio of AM2 AUP shows the opposite. The AM2 peak of Daohuaxiang rice also is at a higher DP than that of Koshihikari rice (Fig. 2b). The $X_{\mathrm{AM} 1}$ values of Daohuaxiang rice and Koshihikari rice are similar; however, the $X_{\mathrm{AM} 2}$ value of Daohuaxiang rice is significantly higher than that of Koshihikari rice. 
Table 1 Parameter values from fitting the biosynthesis model to the amylopectin CLDs of four different rice lines ${ }^{a}$

\begin{tabular}{|c|c|c|c|c|c|c|c|c|c|c|c|c|c|}
\hline Samples & $\beta_{(i)}$ & $\beta_{(\mathrm{ii})}$ & $\beta_{\text {(iii) }}$ & $\beta_{\text {(iv) }}$ & $\beta_{(v)}$ & $\beta_{(\mathrm{vi})}$ & $\gamma_{(i, i, i)}$ & $\gamma_{(i i, i, i v)}$ & $\gamma_{(v, v i)}$ & $h_{(\mathrm{iii} / \mathrm{i})}$ & $h_{(\mathrm{v} / \mathrm{i})}$ & $X_{\mathrm{AM} 1}$ & $X_{\mathrm{AM} 2}$ \\
\hline Wild type & 0.15 & 0.0115 & 0.0659 & 0.00934 & 0.0453 & 0.0000028 & 0.0582 & 0.0507 & 0.0361 & 0.113 & 0.00965 & 920 & 2172 \\
\hline BEI \& BEllb knockout & 0.0831 & 0.0118 & 0.0468 & 0.00642 & 0.0298 & 0.0001 & 0.005 & 0.0355 & 0.027 & 0.202 & 0.0419 & 933 & 2141 \\
\hline Daohuaxiang & 0.0921 & 0.0354 & 0.0527 & 0.0282 & 0.0465 & 0.012 & 0.0585 & 0.055 & 0.047 & 0.0823 & 0.00909 & 675 & 4711 \\
\hline Koshihikari & 0.104 & 0.038 & 0.0485 & 0.0394 & 0.0461 & 0.0298 & 0.0584 & 0.0556 & 0.0489 & 0.115 & 0.00593 & 664 & 1899 \\
\hline
\end{tabular}

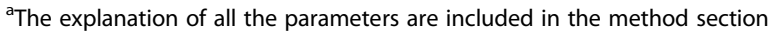

\section{Discussion}

\section{Enzyme activity in amylopectin synthesis}

There are several branching enzyme isoforms in rice grains, including BEI, BEIIa, BEIIb and BEIII (Mizuno et al. 1993). Our results shows that BEI and BEIIb knockout significantly reduces branching ability in rice endosperm: thus BEI and BEIIb knockout WXJ rice have significantly lower amylopectin content than wild type WXJ rice (Fig. 2a) (Zhu et al. 2012). While $\beta_{(\mathrm{i})}, \beta_{\text {(iii) }}$ and $\beta_{\text {(iv) }}$ decrease significantly in the knockouts, the mutations have little effect on $\beta_{\text {(ii) }}$ (Table 1). This result indicates that BEIIa is the main branching enzyme involved in the second enzyme set for amylopectin synthesis $(15<\mathrm{DP}<30)$, while BEI and BEIIb play important roles in the first, third and fourth enzyme sets. BEIIb is essential for the synthesis of short amylopectin chains $(10<\mathrm{DP}<15)$ and BEI is essential for synthesis of long amylopectin chains (DP $>30)$, a result consistent with previous work (Nakamura et al. 2010; Nakamura 2002). The absence of these two enzymes cannot be completely compensated by BEIIa, a result

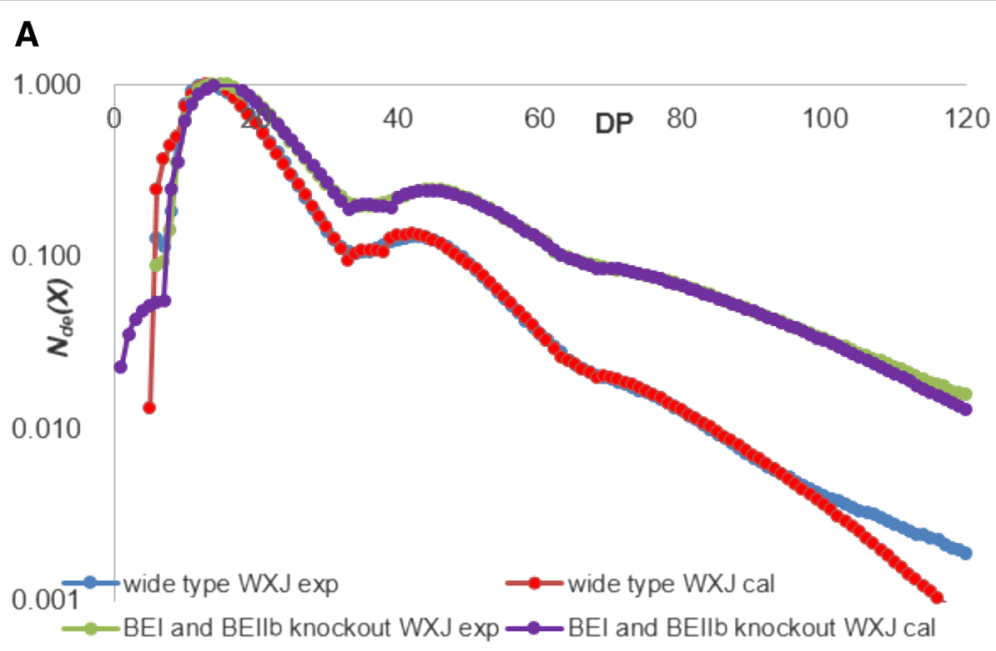

B

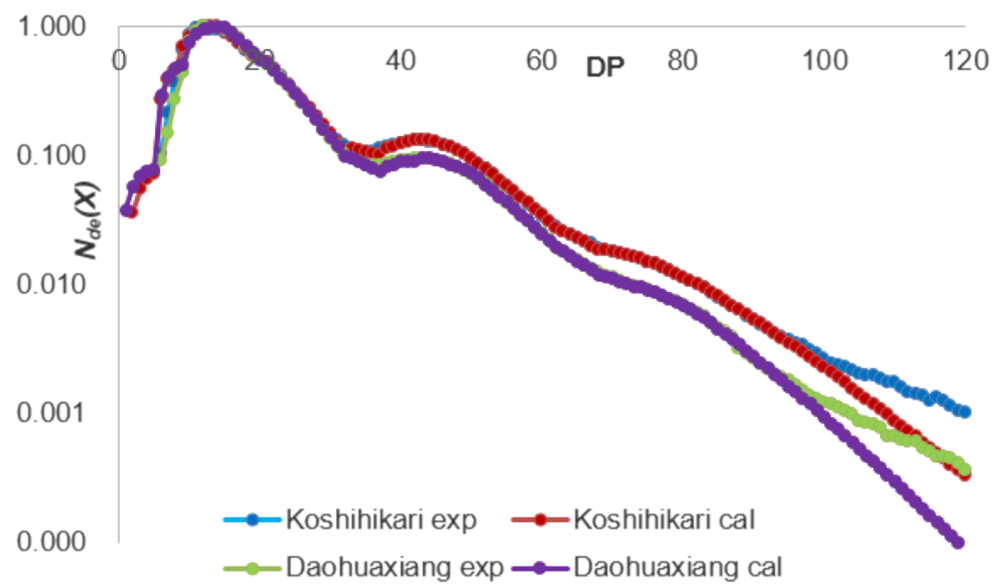

Fig. 1 Experimental ("exp", from FACE) and model-fitted ("cal") number chain-length distributions ( $N_{\text {de }}(X)$, arbitrary normalization) of debranched amylopectin (DP < 100) from wild type and BEl and Bllb knockout rice (a), from Koshihikari and Daohuaxiang (b) 


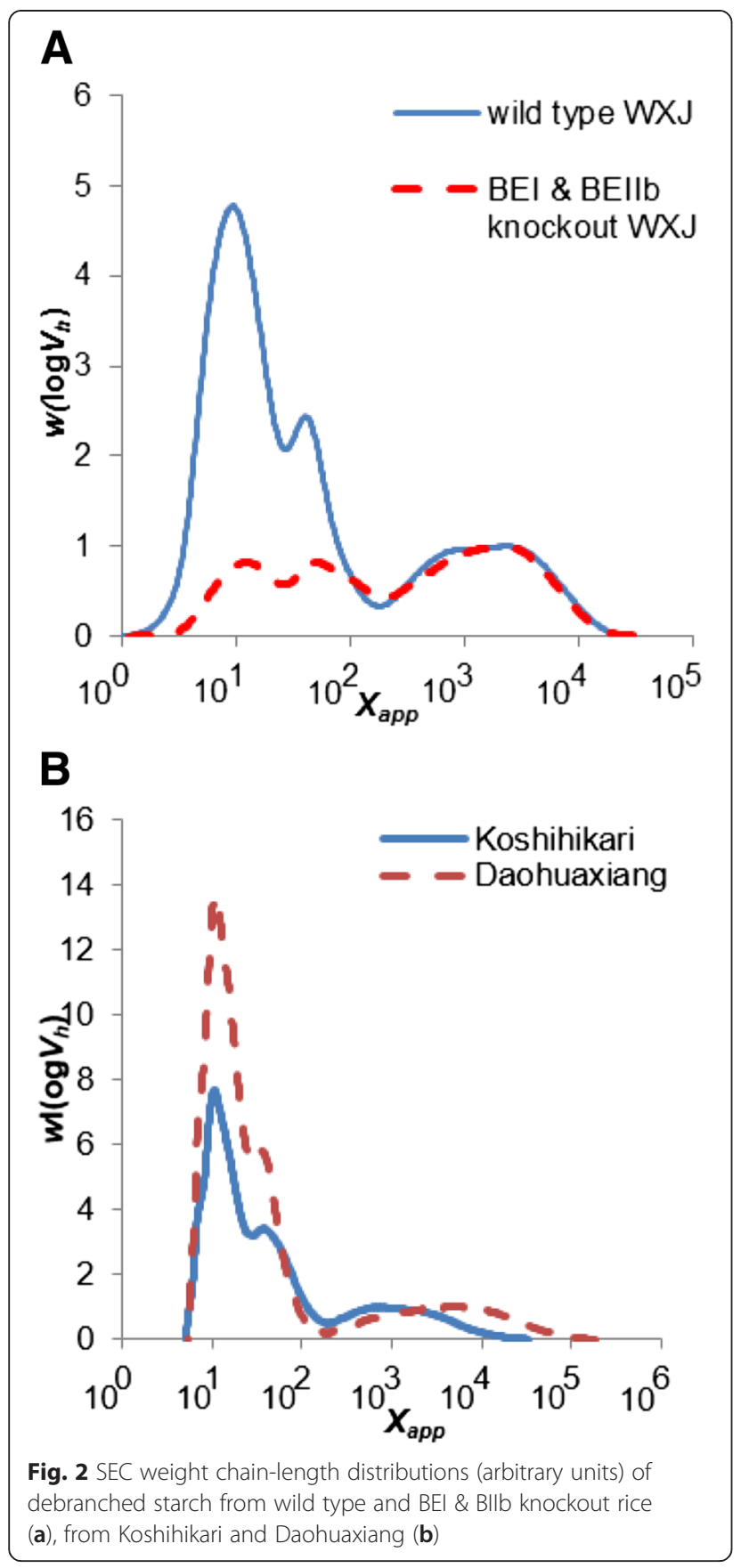

which has not been reported before. Knockout of BEI and BEIIb has more effect on the short amylopectin chains (essentially $\mathrm{A}$ and $\mathrm{B}_{1}$ chains) than on long ones (essentially $\mathrm{B}_{2}$ and $\mathrm{B}_{3}$ chains), as the $h_{(\mathrm{iii} / \mathrm{i})}$ and $h_{(\mathrm{v} / \mathrm{i})}$ values of BEI and BEIIb knockout WXJ rice are larger than those of the wild type. The differences between Daohuaxiang and Koshihikari amylopectin CLDs are not as large as those between wild type WXJ and BEI and BEIIb knockout WXJ rice (Fig. 1 and Table 1), which shows that the difference in enzyme sets in native rice lines is less than that between the wild type and this mutant. The differences in $\beta$ values

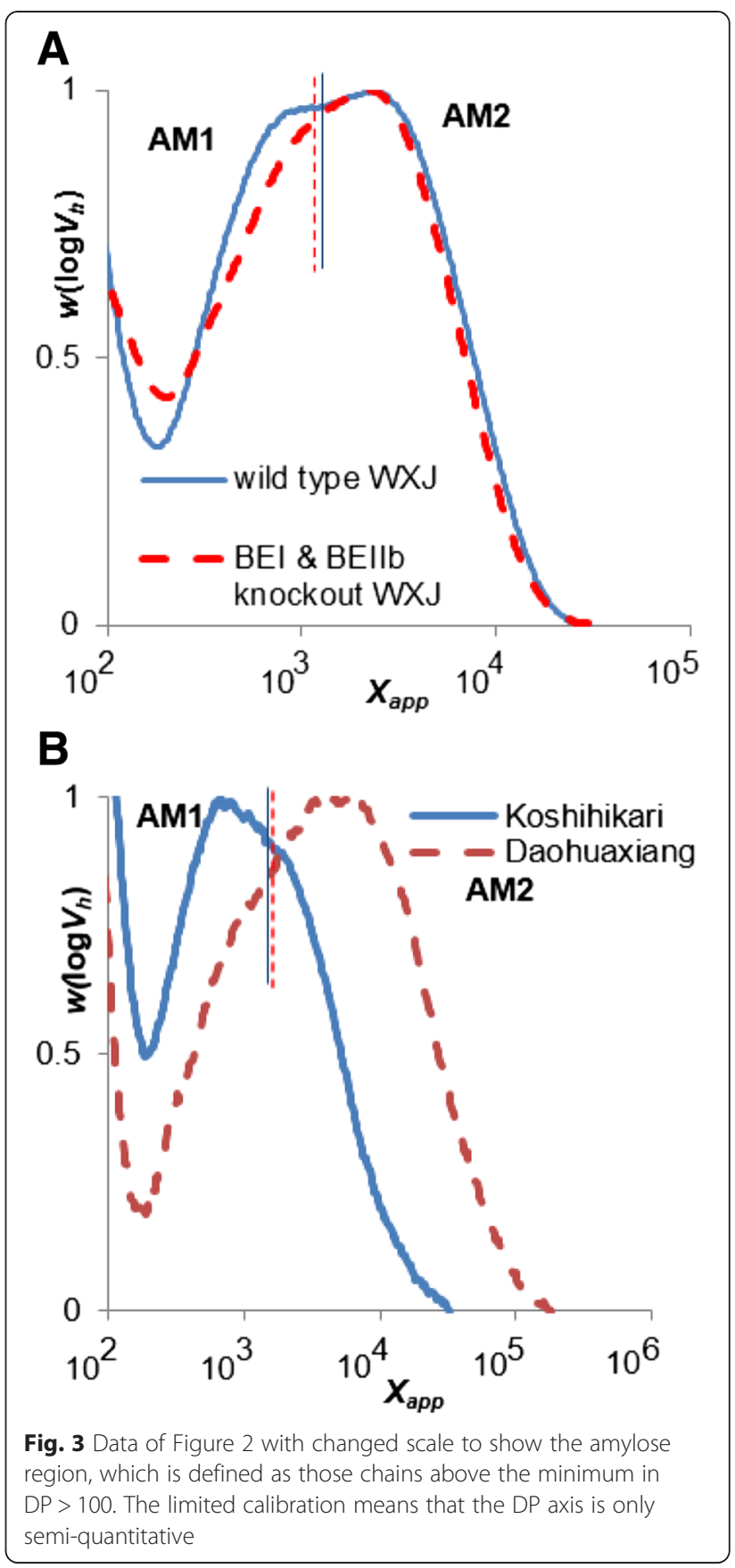

(Table 1) show that different rice lines have different activities for the SBE and SS enzyme sets. However, our results cannot separately distinguish the effects of SBE isoforms and SS isoforms, as the fitting only yields the ratios $\beta$ of their activities.

The values of $\gamma_{(\mathrm{i}, \mathrm{ii})}, \gamma_{(\mathrm{iii}, \mathrm{iv})}$ and $\gamma_{(\mathrm{v}, \mathrm{vi})}$ of wild type WXJ and BEI and BEIIb knockout WXJ decreased in the mutant, although debranching enzyme (DBE) and SS remain unaffected. This is reflected in the BEI and BEIIb knockouts rice grains having less amylopectin: the debranching enzyme (mainly isoamylase, which controls the 
branch spacing to ensure that the CLD is crystallizationcompetent (Wu et al. 2013), and thus the amount of amylopectin (Fujita et al. 2003; Nakamura et al. 1997), works less efficiently. The values of $\gamma_{(\mathrm{i}, \mathrm{ii})}, \gamma_{(\mathrm{iii}, \mathrm{iv})}$ and $\gamma_{(\mathrm{v}, \mathrm{vi})}$ are similar between Daohuaxiang and Koshihikari rice, indicating DBE of those rice varieties work similarly. The $\gamma$ values of the four different rice lines show that only the mutant line has a lower $\gamma$ value, which might indicate that the predicted DBE function can be limited by the amount of its substrates.

The values of $\beta_{(\mathrm{iv})}$ and $\beta_{(\mathrm{vi})}$ of both WXJ samples are much lower than other $\beta$ values, which indicates that enzyme sets iv and vi do not have much function in amylopectin synthesis in WXJ samples. However, the $\beta$ values of Daohuaxiang and Koshihikari rice samples do not show much difference; all the enzyme sets play important roles in amylopectin synthesis.

\section{Enzyme activity in amylose biosynthesis}

The ratio of the AM1 AUP of wild type WXJ is larger than that of BEI and BEIIb knockout WXJ; the shapes of the AM1 CLD are also different for the two samples, although they have similar $X_{\mathrm{AM} 1}$ (Fig. 2). Debranched SEC results from previous studies have shown that the CLD of short amylose chains (AM1) also contains some long amylopectin chains, although the occurrence of band broadening complicates this conclusion (Horibata et al. 2004). BEI and BEIIb knockout WXJ has more long amylopectin chains than wild type WXJ, and thus AM1 of knockout WXJ should have more long amylopectinchain contamination than that of wild type WXJ (Figs. 1, 2 and 3). This indicates that the AM1 AUP difference between knockout and wild type WXJ is not an artifact, and is not due to any long amylopectin chain contamination. The result could suggest that either or both BEI and BEIIb are involved in the synthesis of short amylose chains. In vitro experiments have shown that branching enzymes can catalyze the synthesis of amylose branches (Buleon et al. 1998; Takeda and Hizukuri 1987). BEI has a preference for transferring long amylopectin chains (Nakamura et al. 2010; Mizuno et al. 1993; Nakamura 2002). It could also have a role in transferring short amylose chains, which have been shown to have a similar CLD to those of long amylopectin chains in twodimensional SEC weight distributions (Vilaplana and Gilbert 2010). Although the relative amount of long amylose chains increases in the mutant, the shape of the CLD of long amylose chains and $X_{\mathrm{AM} 2}$ are similar for both samples, which shows that the molecular structure of long amylose chains remains unaffected. This indicates that BEI and BEIIb are probably not involved in the biosynthesis of long amylose chains, although other BEs must be involved to provide a chain-stopping mechanism. There seems to be an association between the higher $\beta(\mathrm{i}), \beta(\mathrm{iv}), \beta(\mathrm{vi})$ and a lower $X_{\mathrm{AM} 2}$ in Koshihikari. The lower $\beta(\mathrm{i}), \beta(\mathrm{iii}), \beta(\mathrm{v})$ values are associated with the BEI and/or BEIIb mutant, which could imply that BEIIa is associated with $\beta$ (ii), $\beta($ vi) and $\beta$ (vi). Together, the above results suggest that BEIIa and/or BEIII might contribute to the shortening of long amylose chains. Further studies on other BE mutants and their amylose CLD could provide evidence for or against this inference.

The shapes of the amylose CLDs, ratios of AM1 AUP and of AM2 AUP and the $X_{\mathrm{AM} 2}$ value of Daohuaxiang rice show clear differences from those of Koshihikari (Fig. $2 \mathrm{~b}$ and Table 1). Both wild type WXJ and Koshihikari have higher $\beta_{\text {(iv) }}$ (Table 1) and larger AUP of AM1 than the other two samples. This could indicate that besides GBSSI, enzymes responsible for synthesis of long amylopectin chains (such as the fourth enzyme set) might also play an important role in amylose synthesis. The samples with lower $h_{(\mathrm{v} / \mathrm{i})}$ value (wild-type WXJ and Koshihikari) have larger ratios of AM1 AUP, which also might indicate that the amount of long amylopectin and short amylose chains are negatively correlated. BEI is one of the enzymes that can affect synthesis of short amylose chains, as discussed above. The effect of SS is not analyzed in this study; however, SS involving long amylopectin chain synthesis might be able to affect short amylose chain synthesis. SSIII, which mainly contributes to the elongation of long inner amylopectin $\left(B_{2}, B_{3}\right)$ chains (Edwards et al. 1999; Lloyd et al. 1999), could be another enzyme involved in synthesis of short amylose chains.

SSS (soluble starch synthase) is unlikely to be involved in the synthesis of long amylose chains, as there is nearly no amylose produced in GBSSI knockout grain (Myers et al. 2000; Denyer et al. 2001). The activity of GBSSI could therefore be responsible for the structural differences in long amylose chains of Daohuaxiang and Koshihikari. There is another possibility: that the AM2 CLD differences are because of pullulanase activity differences. The increased pullulanase activity would break down more uncrystallized amylopectin molecules and thus provide more sugar substrate for long amylose chain synthesis.

\section{Conclusion}

Amylose chains in the wild-type and mutant rice samples examined here can be divided into two different groups, long and short, with different ranges of degrees of polymerization showing distinct features in the chainlength distribution. The present results show that these two amylose groups have different dominant biosynthesis enzymes. As is well known, long amylose chains are mainly synthesized by GBSSI. The synthesis of short amylose chains is found here to be controlled not only by GBSSI but also starch branching enzymes, which are also involved in long amylopectin chain synthesis. 
Because these smaller amylose chains have significant effects on functional properties such as mouth-feel and digestibility (Syahariza et al. 2013; Syahariza et al. 2014), this finding has potential application in the development of improved crop varieties.

\section{Methods \\ Materials}

Two groups of rice samples were used in this work. One group is the japonica cultivar Wuxiangjing 14 (WXJ) (17\% apparent amylose content) and its transgenic line with the BEI \& BEIIb knockout (68\% apparent amylose content) (Zhu et al. 2012). The other group comprises two japonica rice cultivars with good cooking and eating quality, Daohuaxiang and Koshihikari. Both rice cultivars have same type of $W x$ allele $\left(W x^{b}\right)$ with less amylose content $(15-16 \%)$ compared to rice lines with normal $W x^{a}$ allele. All rice lines were grown and harvested on the campus of Yangzhou University in 2012, and their mature seeds were ground into fine flour using a coffee grinder. Starch was extracted from rice flour and debranched using the method of $\mathrm{Li}$ et al. (2011). The debranched starch was freeze-dried overnight and stored in a desiccator prior to structural analysis.

\section{Fluorophore-assisted carbohydrate electrophoresis}

The CLD of debranched amylopectin was analyzed using FACE, using an improved extraction method (Wu et al. 2014). Debranched starch ( 0.3 mg) was incubated with $1.5 \mu \mathrm{L}$ of a solution of $0.2 \mathrm{M}$ APTS (8-amino-1,3,6-pyrene trisulfonic acid, 501309, Beckman-Coulter) in $15 \%$ glacial acetic acid and $1.5 \mu \mathrm{L}$ of $1 \mathrm{M}$ sodium cyanoborohydride in tetrahydrofuran solution (296813, Sigma). The mixture was vortexed and incubated at $60{ }^{\circ} \mathrm{C}$ for $90 \mathrm{~min}$ for fluorophore labeling of the debranched amylopectin chains, following the method described in the Beckman-Coulter carbohydrate labeling and analysis kit. The solution of labeled glucans was diluted by adding $100 \mu \mathrm{L}$ water. The mixture was centrifuged at $4000 \mathrm{~g}$ for $2 \mathrm{~min}$ and $70 \mu \mathrm{L}$ of the supernatant was transferred to PCR tubes for immediate FACE analysis. The analysis was carried on a PA-800 Plus System using a solid-state laser-induced fluorescence detector with an argon-ion laser as the excitation source (Beckman-Coulter, Brea, CA, USA). A $50-\mu \mathrm{m}$ diameter $\mathrm{N}-\mathrm{CHO}$ coated capillary with separation length $\sim 42 \mathrm{~cm}$ and carbohydrate separation buffer (both included in the carbohydrate labeling and analysis kit) were used for separating amylopectin branches. The sample was injected into the capillary by pressure injection for $3 \mathrm{~s}$ at $0.5 \mathrm{psi}$, and the separation of samples was driven with an applied voltage of $30 \mathrm{kV}$ at $25{ }^{\circ} \mathrm{C}$. The whole separation process takes $90 \mathrm{~min}$. The areas of the peaks (which are essentially baseline-separated in FACE) directly correspond to the relative number glucan chains (the number CLD) in the peak.

\section{Fitting of amylopectin CLD using the biosynthesis model}

The CLDs of amylopectin gained from FACE were fitted to a model developed by $\mathrm{Wu}$, Gilbert and colleagues (Wu et al. 2013; Wu and Gilbert 2010), using a publicly available FORTRAN program package (Wu et al. 2013). The basic premise is that the CLD of chains spanning one, two and three lamellae is controlled by different enzyme sets, and that there are two sets per lamella, a total of six sets. The model has a number of parameters. Each enzyme set is parameterized by the ratio of activity of SBE to that of SS $(\beta)$, that of debranching enzyme to that of SS $(\gamma)$, the minimal chain length for an SBE to break an $\alpha-(1 \rightarrow 4)$ linkage and attach the fragment on the same or on another chain to form a new branch $\left(X_{\min }\right)$, and the minimum chain length for the portion remaining of the chain from which this is broken $\left(X_{0}\right)$. Each enzyme set in the six sets is mainly, but by no means exclusively, responsible for the synthesis of branches with a particular range of DP. The first and second enzyme sets are for $A$ and $B_{1}$ chains, the third and fourth enzyme sets are for $B_{2}$ chains, and the fifth and sixth enzyme sets are for $B_{3}$ chains. $\beta_{(\mathrm{i})}, \beta_{(\mathrm{ii})}$ etc. refer to the value of $\beta$ for the first, second, etc., enzyme set. For a given plant species, there are two alternative models describing the interactions between two enzyme sets in a given lamella: the substrate-independent and substrate-competing models. For rice, it is found that the substrate-competing model is sufficient for quantitative fits (Wu et al. 2013). The additional parameters are then $\gamma_{(\mathrm{i}, \mathrm{ii})}$ and $\gamma_{(\mathrm{iii}, \mathrm{iv})}$, which are the sums of the two $\gamma$ values of the first and second, and third and fourth, enzyme sets respectively. It is noted that, for reasons given in detail by Wu et al. (2010; 2013), the values of $\gamma$ are not independent variables for a given enzyme set, but instead are fixed by a zero-eigenvalue relationship from the value of $\beta, X_{\min }$ and $X_{0}$ for each enzyme set. The final parameters for the substrate-competing version of the model are $h_{(\mathrm{i}, \mathrm{iii})}$ and $h_{(\mathrm{i}, \mathrm{v})}$, which respectively are the ratios of the maximum of the CLD component synthesized by the enzyme set iii + iv combined to that of $\mathrm{i}+\mathrm{ii}$ combined, and $\mathrm{v}+\mathrm{vi}$ to that of $\mathrm{i}+\mathrm{ii}$.

Typical amylopectin CLDs show a number of features, including a global maximum in the range of the branches spanning one lamella, another peak or shoulder in the range spanning two lamellae and another in the range spanning three lamellae.

\section{Size exclusion chromatography analysis}

The CLD of debranched starch was analyzed by SEC following the method of Li et al. (2011) Debranched starch $(2 \mathrm{mg})$ was dissolved in DMSO with $0.5 \%(\mathrm{w} / \mathrm{w})$ 
$\mathrm{LiBr}$ for $2 \mathrm{~h}$ at $80^{\circ} \mathrm{C}$. This solution was analyzed with an Agilent 1100 Series SEC system (Agilent Technologies, Waldbronn, Germany) equipped with GRAM 100 and GRAM 1000 analytical columns (Polymer Standards Service (PSS), Mainz, Germany) set at $80{ }^{\circ} \mathrm{C}$ and a differential refractive index (DRI) detector (Wyatt, Santa Barbara, CA, USA). DMSO containing $0.5 \% \mathrm{w} / \mathrm{w} \mathrm{LiBr}$ was used as the eluent and the flow rate was $0.6 \mathrm{~mL} \mathrm{~min}^{-1}$. Calibration was with two pullulan standards of molecular weight $2.35 \times 10^{6}$ and $1.22 \times 10^{6}$; while the small range offered by these standards means that the conversion of elution volume to molecular weight (or DP), implemented by methods given elsewhere (Vilaplana and Gilbert 2010), will be inaccurate, use of even this limited calibration does mean that the data are reproducible. The results were collected and analyzed with Astra software (Wyatt) using a $\mathrm{d} n / \mathrm{d} c$ value of $0.0717 \mathrm{~mL} \mathrm{~g}^{-1}$ (Li et al. 2011; Syahariza et al. 2013).

\section{Abbreviations}

APTS: 8-Aminopyrene-1,3,6-trisulfonic acid, trisodium salt; SBE: Starch branching enzyme; CLD: Chain length distribution; DBE: Debranching enzyme; DP: Degree of polymerization; FACE: Fluorophore-assisted carbohydrate electrophoresis; GBSS: Granule-bound starch synthase; MOS: Malto-oligosaccharide; SEC: Size exclusion chromatography; SS: Starch synthase; WXJ: Wuxiangjing 14.

\section{Competing interests}

The authors declare that they have no competing interests.

\section{Authors' contributions}

RG conceived the overall approach of the work, EL and ACW drafted the manuscript, EL designed the experiment and did FACE measurements, $J L$ did SEC measurements, ACW did FACE data analysis, QL supplied the rice samples, and $\mathrm{QL}$ and RG edited the manuscript. All authors read and approved the final manuscript.

\section{Authors' information}

EL is a postdoctoral research fellow of HuaZhong University of Science and Technology (China), ACW is a postdoctoral research fellow, University of Queensland (Australia), $J$ L is a masters student of Yangzhou University (China), QL is a professor of Yangzhou University (China) and RG is a professor of both Huazhong University of Science and Technology (China) and University of Queensland (Australia).

\section{Acknowledgement}

The authors would like to thank the 1000-Talents Program of the Chinese Foreign Experts Bureau (RGG), a Chinese postdoctoral grant (EL) and an Australian Postgraduate Award (ACW).

\section{Author details}

${ }^{1}$ Tongji School of Pharmacy, Huazhong University of Science and Technology, Wuhan, Hubei 430030, China. ${ }^{2}$ Centre for Nutrition and Food Sciences, Queensland Alliance for Agricultural and Food Innovation, The University of Queensland, Brisbane, QLD 4072, Australia. ${ }^{3}$ Key Laboratory of Plant Functional Genomics of Ministry of Education, College of Agriculture, Yangzhou University, Yangzhou, Jiangsu 225009, China.

Received: 2 April 2015 Accepted: 11 June 2015

Published online: 17 June 2015

\section{References}

Ball SG, van de Wal MHBJ, Visser RGF (1998) Progress in understanding the biosynthesis of amylose. Trends Plant Sci 3(12):462-467

Buleon A, Colonna P, Planchot V, Ball S (1998) Starch granules: structure and biosynthesis. Int J Biological Macromolecules 23(2):85-112
Castro JV, Ward RM, Gilbert RG, Fitzgerald MA (2005) Measurement of the Molecular Weight Distribution of Debranched Starch. Biomacromolecules 6(4):2260-2270

Denyer K, Johnson P, Zeeman SC, Smith AM (2001) The control of amylose synthesis. J Plant Physiol 158:479-487

Edwards A, Fulton DC, Hylton CM, Jobling SA, Gidley M, Rossner U, Martin C, Smith AM (1999) A combined reduction in activity of starch synthases II and III of potato has novel effects on the starch of tubers. Plant J 17(3):251-261

Fujita N, Kubo A, Suh D-S, Wong K-S, Jane J-L, Ozawa K, Takaiwa F, Inaba Y, Nakamura Y (2003) Antisense inhibition of isoamylase alters the structure of amylopectin and the physicochemical properties of starch in rice endosperm. Plant Cell Physiol 44(6):607-618

Hizukuri S (1985) Relationship between the distribution of the chain-length of amylopectin and the crystalline-structure of starch granules. Carbohydr Res 141(2):295-306

Hizukuri S (1986) Polymodal distribution of the chain lengths of amylopectins, and its significance. Carbohydr Res 147(2):342-347

Horibata T, Nakamoto M, Fuwa H, Inouchi N (2004) Structural and Physicochemical Characteristics of Endosperm Starches of Rice Cultivars Recently Bred in Japan. J Appl Glycosci 51(4):303-313

Li E, Hasjim J, Dhital S, Godwin ID, Gilbert RG (2011) Effect of a gibberellin-biosynthesis inhibitor treatment on the physicochemical properties of sorghum starch. J Cereal Sci 53(3):328-334

Lloyd JR, Landschutze V, Kossmann J (1999) Simultaneous antisense inhibition of two starch-synthase isoforms in potato tubers leads to accumulation of grossly modified amylopectin. Biochem J London 338(2):515-521

Mizuno K, Kawasaki T, Shimada H, Satoh H, Kobayashi E, Okumura S, Arai Y, Baba $T$ (1993) Alteration of the structural properties of starch components by the lack of an isoform of starch branching enzyme in rice seeds. J Biol Chem 268(25):19084-19091

Mua JP, Jackson DS (1997) Relationships between Functional Attributes and Molecular Structures of Amylose and Amylopectin Fractions from Corn Starch. J Agric Food Chem 45(10):3848-3854

Myers AM, Morell MK, James MG, Ball SG (2000) Recent progress towards understanding biosynthesis of the amylopectin crystal. Plant Physiol 122(4):989-997

Nakamura Y (2002) Towards a better understanding of the metabolic system for amylopectin biosynthesis in plants: Rice endosperm as a model tissue. Plant Cell Physiol 43(7):718-725

Nakamura Y, Kubo A, Shimamune T, Matsud T, Harada K, Satoh H (1997) Correlation between activities of starch debranching enzyme and alpha-polyglucan structure in endosperms of sugary-1 mutants of rice. Plant J 12(1):143-153

Nakamura Y, Utsumi Y, Sawada T, Aihara S, Utsumi C, Yoshida M, Kitamura S (2010) Characterization of the Reactions of Starch Branching Enzymes from Rice Endosperm. Plant Cell Physiol 51(5):776-794

Syahariza ZA, Sar S, Tizzotti M, Hasjim J, Gilbert RG (2013) The importance of amylose and amylopectin fine structures for starch digestibility in cooked rice grains. Food Chem 136(2):742-749

Syahariza ZA, Sar S, Warren FJ, Zou W, Hasjim J, Tizzotti MJ, Gilbert RG (2014) Corrigendum to "The importance of amylose and amylopectin fine structures for starch digestibility in cooked rice grains" [Food Chem. 136 (2013) 742-749]. Food Chem 145(0):617-618

Takeda Y, Hizukuri S (1987) Structures of rice amylopectins with low and high affinities for iodine. Carbohydr Res 168:79-88

Tester RF, Karkalas J, Qi X (2004) Starch-composition, fine structure and architecture. J Cereal Sci 39(2):151-165

Vilaplana F, Gilbert RG (2010) Two-dimensional size/branch length distributions of a branched polymer. Macromolecules 43(17):7321-7329

Wang LZ, White PJ (1994) Structure and properties of amylose, amylopectin, and intermediate materials of oat starches. Cereal Chem 71(3):263-268

Ward RM, Gao Q, de Bruyn H, Gilbert RG, Fitzgerald MA (2006) Improved methods for the structural analysis of the amylose-rich fraction from rice flour. Biomacromolecules 7(3):866-876

Witt T, Doutch J, Gilbert EP, Gibert RG (2012) Relations between molecular, crystalline, and lamellar structures of amylopectin. Biomacromolecules 13(12):4273-4282

Wu AC, Gilbert RG (2010) Molecular weight distributions of starch branches reveal genetic constraints on biosynthesis. Biomacromolecules 11(12):3539-3547 
Wu AC, Morell MK, Gilbert RG (2013) A parameterized model of amylopectin synthesis provides key insights into the synthesis of granular starch. PLoS ONE 8(6):e65768

Wu AC, Li E, Gilbert RG (2014) Extraction/dissolution procedures for analysis of starch chain-length distributions. Carbohydr Polym 114(1):36-42

Yoshimoto Y, Tashiro J, Takenouchi T, Takeda Y (2000) Molecular structure and some physicochemical properties of high-amylose barley starches. Cereal Chem 77(3):279-285

Zhu L, Gu M, Meng X, Cheung SCK, Yu H, Huang J, Sun Y, Shi Y, Liu Q (2012) High-amylose rice improves indices of animal health in normal and diabetic rats. Plant Biotechnol J 10(3):353-362

Submit your manuscript to a SpringerOpen ${ }^{\circ}$ journal and benefit from:

- Convenient online submission

- Rigorous peer review

- Immediate publication on acceptance

- Open access: articles freely available online

- High visibility within the field

- Retaining the copyright to your article

Submit your next manuscript at $\gg$ springeropen.com 